Ali Mehdi, MD, MEd, FACP

Department of Nephrology and Hypertension,

Glickman Urologic and Kidney Institute, Cleveland

Clinic, Cleveland, $\mathrm{OH}$; Assistant Professor, Cleveland

Clinic Lerner College of Medicine of Case Western

Reserve University, Cleveland, $\mathrm{OH}$
George Thomas, MD

Department of Nephrology and Hypertension,

Glickman Urologic and Kidney Institute, Cleveland

Clinic, Cleveland, OH; Assistant Professor, Cleveland

Clinic Lerner College of Medicine of Case Western

Reserve University, Cleveland, $\mathrm{OH}$

\title{
Our evolving understanding of primary aldosteronism
}

P RIMARY ALDOSTERONISM is the most common endocrine cause of hypertension, and is often associated with treatment-resistant hypertension. ${ }^{1}$ It is characterized by autonomous aldosterone production independent of renin activity, potassium level, or volume status. Its prevalence has been debated, and studies are fraught with limitations, including reliance on tests that do not confirm the diagnosis. It has been identified as a public health issue, ${ }^{2}$ and the urgency in identifying and treating it is heightened by its strong association with adverse kidney and cardiovascular outcomes. ${ }^{1,3,4}$

Multiple studies have pointed to limitations of our current screening strategies, which may be missing a great many patients who have the disease. ${ }^{5,6}$ In particular, newer evidence highlights shortcomings of the longaccepted screening strategy that uses the spot aldosterone-to-renin ratio. ${ }^{7}$

In this commentary, we review the current understanding of primary aldosteronism and the current landscape of screening practices. We will highlight the emerging evidence suggesting that renin-independent hyperaldosteronism is best viewed as a continuum that extends across the spectrum of blood pressure severity. We also describe a proposed approach to optimize case detection of primary aldosteronism.

\section{SERUM POTASSIUM IS NOT ALWAYS LOW}

Primary aldosteronism was first described in 1954 by Dr. Jerome Conn, ${ }^{8}$ who noted a syndrome of periodic muscle weakness, hypokalemia, alkalosis, and hypertension with elevated urinary aldosterone levels.

doi:10.3949/ccjm.88a.20166
Subsequently, hypokalemia came to be viewed as an important component of this condition, to the point that it became almost a sine qua non for the diagnosis. Although primary aldosteronism was classically suspected in patients presenting with the triad of hypertension, hypokalemia, and metabolic alkalosis, this phenotype likely describes an extreme form of the condition. In fact, only $9 \%$ to $37 \%$ of patients with primary aldosteronism have hypokalemia, and the most common presentation is normokalemic hypertension. ${ }^{2}$ Hypokalemia often alerts the clinician to a possible diagnosis of primary aldosteronism; however, the absence of hypokalemia holds a poor negative predictive value for diagnosis and should not be used to exclude the presence of the disorder.

\section{MORE COMMON THAN THOUGHT, BUT TRUE PREVALENCE IS UNCLEAR}

The prevalence of primary aldosteronism varies significantly among published reports, and its true prevalence remains unclear. What is becoming clear, however, is that the old notion that this condition accounts for fewer than $1 \%$ of cases of mild to moderate hypertension is not true. ${ }^{2}$ The variability in the reported prevalence stems primarily from the populations being studied, ie, the general population vs hypertensive patients vs those with resistant hypertension. Most experts now accept that the prevalence of primary aldosteronism is between $5 \%$ and $10 \%$ among all hypertensive patients, and up to $20 \%$ in those with resistant hypertension. ${ }^{9,10}$

A major challenge in studying the epidemiology of primary aldosteronism is that there is no gold standard for diagnosing it. The de-

\section{Primary aldosteronism is not a rare disorder}




\section{TABLE 1}

\section{Positive thresholds of biochemical testing for primary aldosteronism}

\begin{tabular}{|c|c|c|c|}
\hline Screening tests & More conservative & Most widely accepted & More liberal \\
\hline Aldosterone-renin ratio (ng/dL per ng/mL/hour) & $\geq 40$ & $\geq 30$ & $\geq 20$ \\
\hline Plasma aldosterone concentration (ng/dL) & $\geq 20$ & $\geq 15$ & $\geq 10$ \\
\hline Confirmation aldosterone suppression tests & More conserv & tive & More liberal \\
\hline $\begin{array}{l}\text { With oral salt } \\
24-H o u r \text { urinary aldosterone excretion rate }(\mu \mathrm{g})\end{array}$ & $>12-14$ & & $>10$ \\
\hline $\begin{array}{l}\text { With intravenous saline } \\
\text { Plasma aldosterone concentration ( } \mathrm{ng} / \mathrm{dL} \text { ) }\end{array}$ & $>10$ & & $>5$ \\
\hline $\begin{array}{l}\text { With fludrocortisone } \\
\text { Seated plasma aldosterone concentration (ng/dL) }\end{array}$ & $\begin{array}{l}\quad>6 \\
\text { with plasma renir } \\
<1 \mathrm{ng} / \mathrm{mL} / \mathrm{h}\end{array}$ & $\begin{array}{l}\text { activity } \\
\text { ur }\end{array}$ & \\
\hline $\begin{array}{l}\text { With captopril } \\
\text { Plasma aldosterone concentration, } \\
\text { decrease from baseline } \\
\text { Aldosterone-renin ratio (ng/dL per na/mL/hour) }\end{array}$ & $\begin{array}{c}<30 \% \\
>30\end{array}$ & & $>20$ \\
\hline
\end{tabular}

\section{The most \\ common \\ presentation is \\ normokalemic \\ hypertension}

fault standard has been the aldosterone-renin ratio, but the diagnostic cutoff varies across practices and has not been validated in prospective trials. Liberalizing or restricting the diagnostic cutoffs can have significant effects on the perceived prevalence.

\section{CURRENT GUIDELINES FOR SCREENING AND DIAGNOSIS}

\section{First step: Screening with the ratio of aldosterone to renin}

The current Endocrine Society guidelines for detecting and diagnosing primary aldosteronism, published in 2016, recommend the aldosterone-renin ratio as the most reliable screening test. ${ }^{2}$ The reporting should include the plasma aldosterone concentration and the plasma renin activity or direct renin concentration, along with the aldosterone-renin ratio. Patients are at high risk of primary aldosteronism and should be screened for it if they have any of the following:

- Sustained hypertension, with blood pressure above 150/100 $\mathrm{mm} \mathrm{Hg}$ on 3 or more occasions
- Hypertension with blood pressure above $140 / 90 \mathrm{~mm} \mathrm{Hg}$ despite the use of 3 or more medications

- Controlled blood pressure less than 140/90 $\mathrm{mm} \mathrm{Hg}$ on 4 or more medications

- Hypertension with spontaneous or diuretic-induced hypokalemia

- Hypertension with obstructive sleep apnea

- Hypertension with an adrenal mass

- Hypertension with a family history of early-onset hypertension or cerebrovascular accident before age 40

- Hypertension with a first-degree relative diagnosed with primary aldosteronism.

The guidelines acknowledge that the threshold defining a high aldosterone-renin ratio (and thus a positive screen) varies by practice, but the most common one is a ratio of $30 \mathrm{ng} / \mathrm{dL}$ per $\mathrm{ng} / \mathrm{mL} /$ hour or higher with a plasma aldosterone concentration of $15 \mathrm{ng} / \mathrm{dL}$ or higher (Table 1).2,11

In the interest of convenience and automation, many laboratories have switched from the traditional plasma renin activity assay to a direct renin concentration assay. While the 
2 assays generally correlate well with one another, conversion factors vary slightly among different laboratories. A discussion of the advantages and disadvantages of each assay is beyond the scope of this commentary. Practitioners, however, must consult with their laboratory when interpreting direct renin concentration and converting to plasma renin activity.

\section{Second step: Confirmatory testing}

Patients who have a positive (high) result on the aldosterone-renin ratio screening test should then undergo a confirmatory diagnostic test to definitively confirm or exclude primary aldosteronism.

The guidelines describe 4 confirmatory testing procedures: oral sodium suppression, saline infusion suppression, fludrocortisone suppression, and captopril challenge test. All these tests hinge on the premise that aldosterone should normally be suppressed with the above interventions. Persistent aldosterone production would be indicative of autonomous renin-independent aldosteronism, thus confirming primary aldosteronism.

The cutoffs used to define a positive result on a confirmatory test again vary by practice (Table 1). . $^{21}$

The guidelines note an exception to the need for the confirmatory step. Patients with spontaneous hypokalemia, suppressed renin, and a plasma aldosterone concentration higher than $20 \mathrm{ng} / \mathrm{mL}$ on the screening test can be confidently given the diagnosis of primary aldosteronism without confirmatory testing.

After the diagnosis of primary aldosteronism is confirmed, patients proceed with subtype evaluation, including computed tomography of the adrenal glands and, potentially, adrenal venous sampling to further classify the primary aldosteronism as being due to adenoma vs hyperplasia. This information can then guide the therapeutic strategy of surgery (adrenalectomy) vs medical therapy with a mineralocorticoid receptor antagonist. Our commentary is limited to case detection, and we acknowledge that subtype classification and treatment of primary aldosteronism is another area of intense discussion and yet another element in the changing landscape of primary aldosteronism.

\section{LIMITATIONS OF CURRENT STATE}

Current guidelines recommend screening for primary aldosteronism only in patients with relatively severe hypertension. But restricting screening to this population allows less severe and, possibly, early cases to go undetected for many years, if they are ever diagnosed.

Monticone et $\mathrm{al}^{12}$ screened 1,672 patients referred to a hypertension center in Italy. Using relatively conservative thresholds for positive results on screening (aldosterone-renin ratio $>$ $30 \mathrm{ng} / \mathrm{dL}$ per $\mathrm{ng} / \mathrm{mL} /$ hour and a plasma aldosterone concentration $>10 \mathrm{ng} / \mathrm{dL}$ ), confirmatory testing with intravenous saline suppression identified primary aldosteronism in 10\% to $12 \%$ of patients who met the screening criteria. More importantly, $4 \%$ of patients with untreated hypertension (blood pressure 140 159/90-99 mm Hg) were also found to have primary aldosteronism. These patients did not meet any screening criteria and would not have been tested outside a research setting.

Other studies show that even in high-risk groups in which screening is recommended, rates of screening and eventual diagnosis remain unacceptably low. 5,6

The limitations in screening are further confounded by the arbitrary aldosterone-renin ratio cutoffs used. As noted, the most widely accepted threshold for a positive screening test is an aldosterone-renin ratio of $30 \mathrm{ng} / \mathrm{dL}$ per $\mathrm{ng} / \mathrm{mL} /$ hour or higher with a minimum plasma aldosterone concentration of $15 \mathrm{ng} / \mathrm{dL}$ or higher. However, multiple studies have repeatedly demonstrated higher detection rates when the criteria were more relaxed. ${ }^{11}$ In particular, in patients with low plasma renin activity $(<$ $1 \mathrm{ng} / \mathrm{mL} /$ hour), plasma aldosterone concentration cutoffs of $9 \mathrm{ng} / \mathrm{dL},{ }^{2}$ or even as low as $6 \mathrm{ng} /$ $\mathrm{dL},{ }^{13}$ have increased the number of cases detected.

\section{A practice-changing study}

The strongest evidence yet of the limitation of our screening test of choice comes from a study by Brown et al. ${ }^{7}$ The study cohort was derived from 5 study protocols across the United States representing a spectrum of blood pressure phenotypes ranging from normotension to resistant hypertension. A total of 1,846 patients were included in the study.

Blood pressure medications were with-

\section{Even milder forms of renin- independent aldosterone production are probably not benign}


drawn, except in patients with resistant hypertension, in whom medications aside from mineralocorticoid receptor antagonists and epithelial sodium channel inhibitors were continued. Then, all patients underwent oral salt loading for 3 days, after which plasma renin activity, plasma aldosterone concentration, and 24-hour urinary aldosterone excretion were measured. The authors used a conservative threshold aldosterone excretion rate of $12 \mu \mathrm{g} / 24$ hours to define "biochemically overt primary aldosteronism" in the context of high sodium balance and suppressed renin activity.

The rates of primary aldosteronism were high, reaching $11.3 \%$ in normotensive patients. In hypertensive patients, the rates were $15.7 \%$ in those with untreated stage 1 hypertension, $21.6 \%$ in those with untreated stage 2 , and $22 \%$ in those with resistant hypertension. In the subset of patients with suppressed renin, the rates were even higher, reaching $51.6 \%$ in those with resistant hypertension.

However, these rates are perhaps not the most important takeaway from this study. What was remarkable was how poorly the currently accepted screening protocol performed. In patients with confirmed overt primary aldosteronism, the sensitivity (26.9\%) and negative predictive value $(31.8 \%)$ of an aldosterone-renin ratio greater than 30 were low. Even after relaxing the aldosterone-renin ratio cutoff to a more liberal 20 , the sensitivity was a mere $42.3 \%$. Importantly, in the patients with resistant hypertension and confirmed primary aldosteronism, $24.5 \%$ had a plasma aldosterone concentration less than $10 \mathrm{ng} / \mathrm{dL}$ and would have been missed had the standard screening criteria been used.

The authors emphasized the insensitivity of a single aldosterone-renin ratio compared with a 24-hour urine collection, given the known pulsatile nature of aldosterone secretion and the unrecognized fact that adrenocorticotropic hormone is a secretagogue of aldosterone leading to diurnal fluctuations. ${ }^{?}$

It is now clear that the current aldosterone-renin ratio screening test and long-accepted cutoffs are far from being the right answer. In an editorial accompanying the above study, Dr. John Funder ultimately labels the spot measurement of plasma aldosterone con- centration and aldosterone-renin ratio "not merely useless, but actually misleading." 14

\section{THE LOW-RENIN PHENOTYPE}

The limitations in our current understanding do not stop here. The designation of "biochemically overt primary aldosteronism" relies yet again on arbitrary cutoffs for the confirmatory testing (generally accepted as an aldosterone excretion rate of 12 to $14 \mu \mathrm{g} / 24$ hours in the case of the oral salt loading test). ${ }^{2}$ The issue with this definition is that even milder renin-independent aldosterone production that fails to meet these cutoffs might not be entirely benign.

The concept of "nonclassic" or "subclinical" primary aldosteronism has been proposed over the past few years, describing a milder phenotype of dysregulated renin-independent aldosterone production. Indeed, the risk of incident hypertension is reported to be higher in normotensive individuals with a high aldosterone-renin ratio and plasma aldosterone concentration values not meeting the criteria for primary aldosteronism. ${ }^{15} \mathrm{~A}$ follow-up study also showed that the rate of incident hypertension was high in participants with the lowest renin concentrations. ${ }^{16}$ Brown et $\mathrm{al}^{17}$ reported similar outcomes in another cohort, in which suppressed renin activity $(\leq 0.5 \mathrm{ng} / \mathrm{mL} /$ hour) was associated with significantly higher risk of incident hypertension among normotensive participants compared with those with unsuppressed renin. Importantly, incident hypertension correlated positively with the plasma aldosterone concentration even at levels considered to be in the normal range.

Interest in the low-renin profile is not new. In 1969 , Woods et $\mathrm{al}^{18}$ reported that hypertensive patients with the low-renin phenotype responded to adrenal blockade with significant decreases in blood pressure. The clinical significance of this suppressed renin phenotype is further highlighted in the PATHWAY-2 study, ${ }^{19}$ which showed that spironolactone was the most effective add-on medication in resistant hypertension. Importantly, primary aldosteronism was excluded by a specialist before patients entered this study. In a follow-up analysis, ${ }^{20}$ there was favorable blood pressure response to spironolactone when renin was 
suppressed, and the response correlated positively with plasma aldosterone concentration and aldosterone-renin ratio (even within the "normal" range). ${ }^{20}$ This analysis raises the question as to whether this population of patients with resistant hypertension included some with a milder form of primary aldosteronism who simply did not meet the current criteria for diagnosis of the disease.

In data presented at the American Society of Nephrology in 2019 (unpublished), we evaluated 1,142 patients with chronic kidney disease in whom renin and plasma aldosterone concentration values were obtained. After excluding patients with primary aldosteronism, patients with suppressed renin values had more resistant hypertension and faster decline in glomerular filtration rate than those with unsuppressed renin. We speculate that some of these patients with suppressed renin may actually have a milder form of primary aldosteronism.

\section{HYPERALDOSTERONISM AS A SPECTRUM}

Dysregulated aldosterone production is not just a cause of resistant hypertension; even at levels below the currently accepted criteria for primary aldosteronism, it is associated with adverse cardiovascular, metabolic, and kidney outcomes. ${ }^{17,21}$ In the study by Brown et $\mathrm{al}^{7}{ }^{7}$ a clear continuum of renin-independent aldosterone production was observed in each of the 4 blood pressure categories (normotension, stage 1 hypertension, stage 2 hypertension, and resistant hypertension) despite the high sodium balance. As expected, the magnitude of aldosterone production was progressively higher across the blood pressure spectrum. Importantly, a continuous relationship was observed between the magnitude of the renin-independent aldosterone production as well as signs of excess mineralocorticoid activity (systolic and diastolic blood pressure, serum potassium level, and urinary potassiumto-sodium ratio).

Given the above, the authors call for reframing our terminology of primary aldosteronism and the arbitrary binary cutoffs to highlight the spectrum of this condition characterized by renin-independent aldosterone production-one that goes from a subclinical form where patients can be normotensive, to milder clinically significant phenotypes not meeting current diagnostic criteria, and all the way to an overt phenotype of severe hypertension and hypokalemia. ${ }^{11}$

\section{WHERE DO WE GO FROM HERE?}

Current screening practices likely capture only a small portion of the more severe phenotype of primary aldosteronism. Given the potential cardiometabolic and kidney implications of even the milder forms of primary aldosteronism, the current guidelines need to be reexamined. This is particularly important, as these risks are potentially modifiable with targeted therapy with mineralocorticoid receptor antagonists. In Figure 1, we propose an approach to screening and diagnosing primary aldosteronism.

New practice guidelines will, hopefully, address the following issues:

Greater awareness in the general medical community is needed to emphasize that primary aldosteronism is not a rare disorder and that it is particularly common in the high-risk population in which screening is classically recommended.

Identifying populations to screen other than the classic high-risk populations. This decision will have to balance the cost-effectiveness of expanding screening practices against the risks of missing cases of primary aldosteronism with associated adverse implications. Particular attention should at least be given to severe but not resistant hypertension, hypertension at a young age, and hypertension with concomitant early-onset cardiovascular disease or atrial fibrillation.

Less reliance on the aldosterone-renin ratio. Clearly, the current screening test with single spot aldosterone-renin ratio testing along with the accepted threshold is misleading. At the minimum, the classic high-risk populations should forgo spot testing and pursue confirmatory dynamic testing directly (with oral salt loading, for example).

Looser screening cutoffs. For screening outside the classic high-risk groups, the cutoffs for a positive screen for both the aldosteronerenin ratio and the plasma aldosterone concentration should be liberalized. Given the diurnal variation in aldosterone production,

\section{Much more screening for primary aldosteronism is needed}


Indications to screen for primary aldosteronism

Classic high-risk populations (proceed directly with dynamic confirmatory testing) - - - - ר Hypertension requiring 3 or more blood pressure medications

(controlled or uncontrolled)

Early-onset hypertension (age < 40)

Hypertension and atrial fibrillation without structural heart disease

Hypertension with early-onset cardiovascular disease (age < 40)

Renin not suppressed Plasma aldosterone concentration $<10 \mathrm{ng} / \mathrm{dL}$

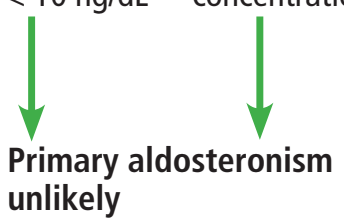

Clinicians should have a low threshold to pursue confirmatory testing, given the low sensitivity of spot screening

${ }^{a}$ Given diurnal variation in plasma aldosterone concentration, repeat testing is suggested in all situations with suppressed renin activity.

Figure 1. Our suggested algorithm for screening for and diagnosis of primary aldosteronism.

repeat screening should be strongly considered in some patients, particularly those with a lowrenin phenotype. ${ }^{22}$ Clinicians should also have a low threshold to pursue confirmatory testing, given the low sensitivity of spot screening.

Streamlined testing. Whether these expanded populations being screened should also bypass the screening test and proceed directly to confirmatory testing will also need to be considered. Cost-effectiveness analyses will be needed.

Looser confirmatory cutoffs. The thresholds for positive confirmatory testing will have to be liberalized as well, particularly in the context of suppressed renin.

New diagnostic tools. Results of evaluation of novel biomarkers of aldosterone excess and mineralocorticoid receptor activation including urinary exosomes and steroid metabolome profiling are awaited. ${ }^{11}$

\section{EVOLVING UNDERSTANDING}

Our understanding of primary aldosteronism has changed dramatically over the past few years. Formerly seen as a rare syndrome 
of resistant hypertension and hypokalemia, primary aldosteronism should now be viewed as a spectrum of autonomous renin-independent aldosterone production that is prevalent across the continuum of blood pressure severity. This condition is associated with higher cardiovascular, metabolic, and renal morbidity, even in its milder phenotypes.

Current screening and diagnostic guide- lines capture only a fraction of the more severe forms of primary aldosteronism and with very poor overall sensitivity. A revamping of current practice guidelines is needed, informed by our newer understanding of this disorder.

\section{DISCLOSURES}

The authors report no relevant financial relationships which, in the context of their contributions, could be perceived as a potential conflict of interest.

\section{REFERENCES}

1. Monticone S, D'Ascenzo F, Moretti C, et al. Cardiovascular events and target organ damage in primary aldosteronism compared with essential hypertension: a systematic review and meta-analysis. Lancet Diabetes Endocrinol 2018; 6(1):41-50. doi:10.1016/S2213-8587(17)30319-4

2. Funder JW, Carey RM, Mantero F, et al. The management of primary aldosteronism: case detection, diagnosis, and treatment: an Endocrine Society clinical practice guideline. J Clin Endocrinol Metab 2016; 101(5):1889-1916. doi:10.1210/jc.2015-4061

3. Sechi LA, Novello M, Lapenna R, et al. Long-term renal outcomes in patients with primary aldosteronism. JAMA 2006; 295(22):26382645. doi:10.1001/jama.295.22.2638

4. Hundemer GL, Curhan GC, Yozamp N, Wang M, Vaidya A. Cardiometabolic outcomes and mortality in medically treated primary aldosteronism: a retrospective cohort study. Lancet Diabetes Endocrinol 2018; 6(1):51-59. doi:10.1016/S2213-8587(17)30367-4

5. Jaffe G, Gray Z, Krishnan G, et al. Screening rates for primary aldosteronism in resistant hypertension: a cohort study. Hypertension 2020; 75(3):650-659. doi:10.1161/HYPERTENSIONAHA.119.14359

6. Ruhle BC, White MG, Alsafran S, Kaplan EL, Angelos P, Grogan RH. Keeping primary aldosteronism in mind: deficiencies in screening at-risk hypertensives. Surgery 2019; 165(1):221-227. doi:10.1016/j.surg.2018.05.085

7. Brown JM, Siddiqui M, Calhoun DA, et al. The unrecognized prevalence of primary aldosteronism: a cross-sectional study. Ann Intern Med 2020; 173(1):10-20. doi:10.7326/M20-0065

8. Conn JW. Presidential address. I. Painting background. II. Primary aldosteronism, a new clinical syndrome. J Lab Clin Med 1955; 45(1):3-17. pmid:13233623

9. Hannemann A, Wallaschofski H. Prevalence of primary aldosteronism in patient's cohorts and in population-based studies - a review of the current literature. Horm Metab Res 2012; 44(3):157-162. doi:10.1055/s-0031-1295438

10. Douma S, Petidis K, Doumas M, et al. Prevalence of primary hyperaldosteronism in resistant hypertension: a retrospective observational study. Lancet 2008; 371(9628):1921-1926. doi:10.1016/S0140-6736(08)60834-X

11. Vaidya A, Mulatero P, Baudrand R, Adler GK. The expanding spectrum of primary aldosteronism: implications for diagnosis, pathogenesis, and treatment. Endocrine Rev 2018; 39(6):1057-1088. doi:10.1210/er.2018-00139

12. Monticone S, Burrello J, Tizzani D, et al. Prevalence and clini- cal manifestations of primary aldosteronism encountered in primary care practice. J Am Coll Cardiol 2017; 69(14):1811-1820. doi:10.1016/j.jacc.2017.01.052

13. Stowasser M, Ahmed AH, Pimenta E, Taylor PJ, Gordon RD. Factors affecting the aldosterone/renin ratio. Horm Metab Res 2012; 44(3):170-176. doi:10.1055/s-0031-1295460

14. Funder JW. Primary aldosteronism: at the tipping point. Ann Intern Med 2020; 173(1):65-66. doi:10.7326/M20-1758

15. Vasan RS, Evans JC, Larson MG, et al. Serum aldosterone and the incidence of hypertension in nonhypertensive persons. N Engl J Med 2004; 351(1):33-41. doi:10.1056/NEJMoa033263.

16. Newton-Cheh C, Guo CY, Gona P, et al. Clinical and genetic correlates of aldosterone-to-renin ratio and relations to blood pressure in a community sample. Hypertension 2007; 49(4):846-856. doi:10.1161/01.HYP.0000258554.87444.91

17. Brown JM, Robinson-Cohen C, Luque-Fernandez MA, et al. The spectrum of subclinical primary aldosteronism and incident hypertension: a cohort study. Ann Intern Med 2017; 167(9):630-641. doi:10.7326/M17-0882

18. Woods JW, Liddle GW, Michelakis AM, Brill AB. Effect of an adrenal inhibitor in hypertensive patients with suppressed renin. Arch Intern Med 1969; 123(4):366-370. pmid:5778117

19. Williams B, Macdonald TM, Morant S, et al. Spironolactone versus placebo, bisoprolol, and doxazosin to determine the optimal treatment for drug-resistant hypertension (PATHWAY-2): a randomised, double-blind, crossover trial. Lancet 2015; 386(10008):2059-2068. doi:10.1016/S0140-6736(15)00257-3

20. Williams B, MacDonald TM, Morant SV, et al. Endocrine and haemodynamic changes in resistant hypertension, and blood pressure responses to spironolactone or amiloride: the PATHWAY-2 mechanisms substudies. Lancet Diabetes Endocrinol 2018; 6(6):464-475. doi:10.1016/S2213-8587(18)30071-8

21. Vecchiola A, Fuentes CA, Barros ER, et al. The aldosterone/renin ratio predicts cardiometabolic disorders in subjects without classic primary aldosteronism. Am J Hypertens 2019; 32(5):468-475. doi:10.1093/ajh/hpz023

22. Markou A, Sertedaki A, Kaltsas G, et al. Stress-induced aldosterone hyper-secretion in a substantial subset of patients with essential hypertension. J Clin Endocrinol Metab 2015; 100(8):2857-2864. doi:10.1210/jc.2015-1268

Address: Ali Mehdi, MD, Department of Nephrology and Hypertension, M2, Cleveland Clinic, 9500 Euclid Avenue, Cleveland, OH 44195 ; mehdia@ccf.org 